\title{
Tuning the structure of non-equilibrium soft materials by varying the thermodynamic driving force for crystal ordering $\dagger$
}

\author{
György Tegze, ${ }^{* a}$ László Gránásy, ${ }^{* a b}$ Gyula I. Tóth, ${ }^{a}$ Jack F. Douglas ${ }^{* c}$ and Tamás Pusztai ${ }^{a}$
}

Received 8th September 2010, Accepted 8th November 2010
DOI: 10.1039/c0sm00944j

The present work explores the ubiquitous morphological changes in crystallizing systems with increasing thermodynamic driving force based on a novel dynamic density functional theory. A colloidal 'soft' material is chosen as a model system for our investigation since there are careful colloidal crystallization observations at a particle scale resolution for comparison, which allows for a direct verification of our simulation predictions. We particularly focus on a theoretically unanticipated, and generic, morphological transition leading to progressively irregular-shaped single crystals in both colloidal and polymeric materials with an increasing thermodynamic driving force. Our simulation method significantly extends previous 'phase field' simulations by incorporating a minimal description of the 'atomic' structure of the material, while allowing simultaneously for a description of large scale crystal growth. We discover a 'fast' mode of crystal growth at high driving force, suggested before in experimental colloidal crystallization studies, and find that the coupling of this crystal mode to the well-understood 'diffusive' or 'slow' crystal growth mode (giving rise to symmetric crystal growth mode and dendritic crystallization as in snowflakes by the Mullins-Sekerka instability) can greatly affect the crystal morphology at high thermodynamic driving force. In particular, an understanding of this interplay between these fast and slow crystal growth modes allows us to describe basic crystallization morphologies seen in both colloidal suspensions with increasing particle concentration and crystallizing polymer films with decreasing temperature: compact symmetric crystals, dendritic crystals, fractal-like structures, and then a return to compact symmetric single crystal growth again.

\section{Introduction}

The formation of diverse 'soft' materials composed of synthetic and biological polymers, small molecule 'gelator' molecules, as well as colloid particles and nanoparticles frequently involves structures formed by crystallization under far from equilibrium conditions so that the study of non-equilibrium crystallization lies at the heart of soft matter physics. A truly amazing diversity of structures can be formed in this way, and an equally diverse range of physical properties can be obtained by varying the thermodynamic ordering conditions; a fundamental theoretical and experimental challenge in this field is then to learn how to harness this structural polymorphism to create functional materials. The potential of this approach to material science fabrication is evidenced by numerous examples in the biological world (abalone, the exoskeleton of insects, etc.) where truly remarkable and tuneable (even locally tuneable) material properties can be achieved using essentially a single ordering molecule. There are evident ramifications of this capacity to tailor material properties in relation to the search for advanced materials for diverse applications.

${ }^{a}$ Research Institute for Solid State Physics and Optics, P.O. Box 49, H-1525 Budapest, Hungary.E-mail: turpi@szfki.hu; grana@szfki.hu

${ }^{b}$ Brunel Centre for Advanced Solidification Technology, Brunel University, Uxbridge, Middlesex, UB8 3PH, UK

'Polymers Division, National Institute of Standards and Technology, Gaithersburg, MD, 20899,USA. E-mail: jack.douglas@nist.gov

$\uparrow$ Electronic supplementary information (ESI) available: animations for Fig. 1(b)-(d), 7(a), (b) and 9. See DOI: 10.1039/c0sm00944j
Increasing the thermodynamic driving force for crystallization is normally thought to involve a progressive change in crystal morphology from symmetric single crystal growth at 'near equilibrium' or a low thermodynamic driving force to the formation of symmetric dendrites at an intermediate driving force, followed by polycrystalline spherulitic solidification at a high driving force. ${ }^{1}$ However, recent work has indicated a theoretically unanticipated morphological transition between symmetric and disordered dendrites ('seaweed') in the intermediate driving force regime where the growth patterns remain single crystals. ${ }^{2,3}$ In particular, this cooling-induced change in crystal morphology in near twodimensional polymer films was rationalized by hypothesizing a progressive reduction in the anisotropy of the interfacial free energy upon cooling. ${ }^{4}$ We address this phenomenon computationally using a relatively simple dynamical density functional theory that allows for a description of large scale crystal growth, while accounting for the molecular scale of the crystallizing species. Our simulations indeed indicate that increasing the thermodynamic driving force gives rise to an effective reduction of the growth anisotropy, which we explain as arising from a dynamic broadening of the interface accompanying a competition between diffusive and non-diffusive crystal growth modes. Moreover, the interplay between these growth modes allows us to also describe basic crystallization morphologies seen in colloidal suspensions for increasing particle concentration: compact symmetric crystals, dendritic crystals, fractal-like structures, and then a return to compact symmetric single crystal growth again.

The key to our exploratory computational study of the evolution of crystal structure and growth dynamics upon varying 
the thermodynamic driving force is the adaptation of a minimal field theoretic model of crystalline solidification that incorporates the discrete particle structure of the crystallizing species, represented by periodic solutions of a time-averaged particle density field, whose evolution is governed by diffusion, and an appropriate free energy governing the ordering process. For simplicity, we focus on the crystallization of spherical particles and colloidal measurements, ${ }^{5}$ although many of the general trends are expected to be broadly applicable to other fluids, even polymeric materials, as discussed below.

Numerous theoretical methods have been previously introduced to describe colloidal crystal growth, including adaptations ${ }^{6,7}$ of the classical Wilson-Frenkel (WF) type crystal growth model, cluster dynamics, ${ }^{8}$ a simple field theoretical model, ${ }^{9}$ and density functional theory (DFT). ${ }^{10,11}$ Using a WF based approach, ${ }^{6}$ Russel et $a l^{7}$ have identified some essential patterns in the kinetics of crystal growth that any theory should reproduce (i) a diffusion-controlled or 'slow' growth mode that can account for the three dimensional (3D) dendritic structures observed in experiments on monodisperse colloids in microgravity ${ }^{7,12}$ and (ii) a 'fast' growth mode in which solidification takes place without an appreciable density change. A transition from (i) type growth towards (ii) with increasing supersaturation has also been observed in a simplified field theory that relies on a (nonconserved) structural order parameter coupled to a diffusive (conserved) density field. ${ }^{9}$ The classical DFT, which is a true microscopic theory, is able to predict crystal structure, elasticity, etc. A recent extension of the DFT, the dynamical density functional theory (DDFT), has been used to address crystallization kinetics in 2D. ${ }^{11}$ Past DDFT computations have been limited to only a few hundred particles so that the potential of this promising method has not yet been tested.

We extend DDFT type simulations to a system size $(\sim 13000$ particles) sufficient to address the structures encountered in twodimensional colloidal crystal aggregation., ${ }^{53-15}$ This development is based on an adaptation of the phase-field crystal (PFC) method of Elder et al., ${ }^{16,17}$ an approach that relies on a relatively simple free energy functional. We show below that this new method is able to reproduce the full range of morphologies observed in Skjeltorp's colloidal crystallization experiments, where the driving force for crystallization was varied by increasing the particle concentration. ${ }^{5}$ We find that we are able to interpret these measurements in terms of changes in the growth mechanisms identified in our simulations. Morphology changes at a fixed particle concentration where the temperature is lowered are also considered to explore how increasing driving force through progressive undercooling affects crystal morphology. We again find that increasing the crystallization driving force leads to a progressive change in crystal morphology from symmetric crystal growth to symmetric dendritic growth, and finally to more disordered dendritic growth. This alternative mode of changing crystal morphology provides insights into previous crystallization measurements in thin polymer films ${ }^{2}$ and phase-field simulations of crystal growth intended to model these phenomena. ${ }^{1}$

\section{The phase-field crystal (PFC) model}

The PFC model is a DDFT type approach that relies on timeaveraged particle densities. Starting from the Ramakrishnan-
Yussouff free energy functional, ${ }^{18}$ and performing specific simplifications, one arrives to the familiar Brazovskii/SwiftHohenberg ${ }^{19,20}$ form of the free energy of ordering systems,

$$
F=\int \mathrm{d} r\left\{\frac{\psi}{2}\left[\varepsilon+\left(1+\nabla^{2}\right)^{2}\right] \psi+\frac{\psi^{4}}{4}\right\},
$$

that exhibits a characteristic length scale, which in the present context corresponds to an energetic preference for a well-defined inter-particle distance. In this model, $\varepsilon$ defines the temperature relative to the critical temperature, while $\psi=\left(\rho-\rho_{\mathrm{L}}{ }^{\text {ref }}\right) / \rho_{\mathrm{L}}{ }^{\text {ref }} /$ $\left(3 B_{\mathrm{S}}\right)^{1 / 2}$ is the reduced density of colloidal particles, defined relative to a reference liquid of particle density $\rho_{\mathrm{L}}{ }^{\text {ref }}$. Here $B_{\mathrm{S}}=K /$ $\left(\rho_{\mathrm{L}}{ }^{\text {ref }} k_{\mathrm{B}} T\right)$, while $K$ is the bulk modulus of the crystal, $k_{\mathrm{B}}$ the Boltzmann's constant, and $T$ the temperature. (The term 'particle density' is used here for the surface density of colloidal particles in the two-dimensional system: $\rho=$ number of particles per unit area in our two dimensional simulations.)

To model the time evolution of crystal growth, we follow a DDFT approach previously applied to colloidal systems, ${ }^{11}$ and adopt a diffusive equation of motion with a mobility $M_{\rho}=\rho_{0} D /$ $\left(k_{\mathrm{B}} T\right)$, where $D$ is the self-diffusion coefficient of the particles and $\rho_{0}$ is the particle density in the initial liquid. In dimensionless form:

$$
\frac{\partial \psi}{\partial \tau}=\nabla^{2} \frac{\delta F}{\delta \psi}+\zeta,
$$

where $\tau=t B_{\mathrm{S}}\left[1+\left(\rho_{0}-\rho_{\mathrm{L}}{ }^{\text {ref }}\right) / \rho_{\mathrm{L}}{ }^{\text {ref }}\right] D / R^{2}$ is the dimensionless time scaled with the mobility specified above, whereas $t$ is the dimensional time and $R$ is the size-scale (see Appendix A). Here $\zeta$ stands for a conserved noise characterized by the fluctuationdissipation correlator $\left\langle\zeta(\boldsymbol{r}, \tau) \zeta\left(\boldsymbol{r}^{\prime}, \tau^{\prime}\right)\right\rangle=-\alpha \nabla^{2} \delta\left(\boldsymbol{r}-\boldsymbol{r}^{\prime}\right) \delta\left(\tau-\tau^{\prime}\right)$, where $\alpha$ controls the magnitude of the thermal fluctuations relative to the free energy barriers. ${ }^{21}$ This type of equation of motion is expected to be a good approximation in colloidal suspensions where particle density relaxes diffusively via the Brownian motion of the particles.

The equation of motion has been solved numerically in a parallel environment on a rectangular grid by a semi-implicit fully spectral algorithm (see ref. 22), which is based on an operator-splitting technique. Periodic boundary conditions have been used at the perimeter of the rectangular simulation domains. Details about the choice of material parameters and the conditions under which the computations are performed are explained in Appendix A.

The PFC model at a fixed temperature involves two basic parameters, $\varepsilon$ and $\alpha$, that are related to the compressibility of the liquid, the bulk modulus of the crystal, the diameter of the particles and the density $\rho_{\mathrm{L}}{ }^{\text {ref }}$ of the reference liquid. ${ }^{23}$ The solution also depends on the initial particle density $\psi_{0}$ of the fluid. This model has been used successfully to address elasticity and grain boundaries, ${ }^{16}$ the anisotropies of interfacial free energies, ${ }^{24-26}$ dendritic and eutectic growth, ${ }^{22,27,28}$ glass formation, ${ }^{21}$ polymorphism, ${ }^{29,30}$ heteroepitaxy, ${ }^{27,29}$ and colloidal patterning, ${ }^{23}$ among others. While it is a microscopic approach, it has the advantage over other classical microscopic techniques, such as molecular dynamics simulations, that the time evolution of the system can be studied on the many orders of magnitude longer diffusive timescale, so that the long-time behaviour and largescale crystalline structures become accessible. 
The PFC method has been used recently to model colloidal crystal aggregation and patterning in $2 \mathrm{D}$ and $3 \mathrm{D} .{ }^{23,30}$ It predicts a first-order transition between the homogeneous liquid and the 2D hexagonal structure ${ }^{17,23}$ (see the phase diagram in Fig. 6). Our simulations indicate that melting takes place by vacancy generation and/or grain boundary melting (see Fig. 7). The freezing after quenching at low supersaturations happens either via direct crystal nucleation from the liquid and subsequent growth (see Fig. 8 and 9) or via crystallization from an amorphous precursor at large supersaturations. ${ }^{23}$ These findings are in a remarkable agreement with analogous experiments on colloidal systems, ${ }^{31,32}$ where the appearance of the hexatic phase (of short-range atomic order and quasi-long-range orientational order) predicted by the Kosterlitz-Thouless-Nelson-Halperin-Young theory ${ }^{33}$ could not be observed. This may have several reasons (finite-size effect, hard-core potential, etc.; see discussion in Appendix C), nevertheless, the model and experiment seem to be in a reasonable agreement.

\section{Results and discussion}

\section{Morphology vs. driving force}

We first illustrate the nature of the ordering process the PFC model predicts and then focus on understanding the physical causes of the morphology changes seen when progressively increasing the thermodynamic driving force. The neglect of thermal fluctuations results in the growth of crystals with a perfect six-fold symmetry, but the introduction of noise into the equation of motion breaks this symmetry leading to a randomization in the growth morphology. Representative single-crystal structures obtained by free growth with noise associated with thermal fluctuations $\left(\alpha=10^{-2}\right)$ are shown as a function of the initial reduced particle density of the liquid in Fig. 1. The crystal growth was initiated through introducing a seed (a small hexagonal cluster of seven particles at the centre of the simulation box) and the driving force for the crystalline ordering transition was varied by changing the initial particle density of particles in the fluid. The images in Fig. 1 clearly indicate that increasing the supersaturation [defined here as $S=\left(\psi_{0}-\psi_{\mathrm{L}}{ }^{\mathrm{e}}\right) /$ $\left(\psi_{\mathrm{S}}^{\mathrm{e}}-\psi_{\mathrm{L}}^{\mathrm{e}}\right)$, where $\psi_{\mathrm{S}}{ }^{\mathrm{e}}$ and $\psi_{\mathrm{L}}{ }^{\mathrm{e}}$ are the reduced particle densities of the equilibrium solid and liquid phases, respectively] at first gives rise to slowly growing faceted hexagonal crystals, followed by dendritic and fractal-like crystal patterns, and then more irregular or "porous" crystals form at still high particle densities. We emphasize that each of the patterns exhibits long range order within these crystals. These are crystals rather than polycrystals. As mentioned previously, this pattern of behaviour has been observed in numerous measurements on crystal growth with increasing driving force and we examine the origin of this progressive morphology change in our model. [We note that Fig. 1(a)-(d) show the spatial distribution of the coarse-grained particle density, $\tilde{\psi}$ (i.e., the locally averaged particle density), which has been obtained by two-dimensional FIR (Finite Impulse Response) filtering, ${ }^{34}$ the solution $\psi(\boldsymbol{r})$ of eqn (2). $\tilde{\psi}$ is the analogue of the coarse-grained density used in conventional field theoretic models of crystallization. ${ }^{9}$ Due to coarse-graining, the peaks of $\psi(\boldsymbol{r})$, which represent the individual particles in the crystalline region, cannot be seen in these $\tilde{\psi}$ maps.]

One can expect on general grounds that this progressive morphology change has something to do with some change in the interfacial dynamics induced by the increase in the thermodynamic driving force of crystallization. We next turn off the noise (set $\alpha=0$ in our calculations) so that we can focus on the essential growth modes involved. Noise-free crystal growth morphologies arising from our model are illustrated in Fig. 2. With increasing supersaturation, we first observe the formation of slowly growing faceted hexagonal crystals (Fig. 2a), corresponding to growth under near-equilibrium conditions. This morphology is clearly apparent in the growth pattern with noise (see Fig. 1). This regime is followed by faceted six-fold dendrites

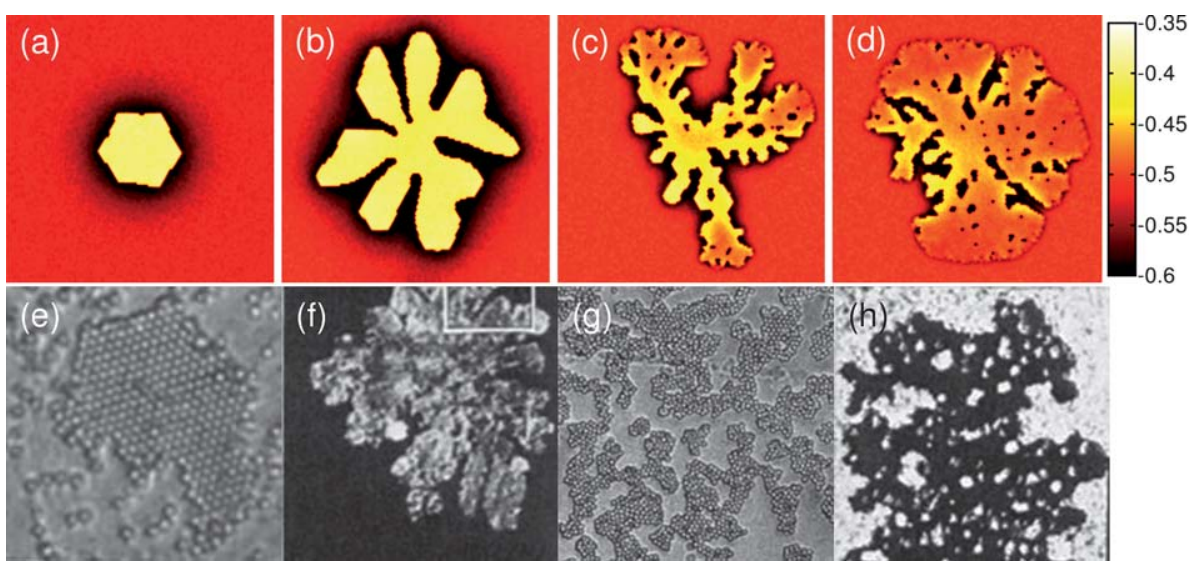

Fig. 1 Single crystal growth morphologies in the presence of noise ((a-d) $\left(\alpha=10^{-2}\right)$ the PFC theory (top) and experiment (bottom) (e-h): 2D colloid crystals by Skjeltorp, ${ }^{5}$ with the permission of the APS; (c) APS 1987). The driving force increases from left to right (in the simulations: $\psi_{0}=-0.52$; $-0.505 ;-0.504$; and -0.5035$)$. In the case of the simulations, the coarse-grained particle density map (obtained by $2 \mathrm{D}$ FIR filtering, see text) is shown. The fractal dimensions of the single crystal aggregates evaluated from the slope of the plot $\log (N) v s \cdot \log \left(R_{\mathrm{g}}\right)$ ( $N$ is the number of particles in the cluster and $R_{\mathrm{g}}$ is its radius of gyration) are: (a) $f_{\mathrm{d}}=2.012 \pm 0.3 \%$; (b) $1.967 \pm 0.3 \%$; (c) $1.536 \pm 0.9 \%$; (d) $1.895 \pm 0.3 \%$. The error is the standard deviation of the slope. The fast growth mode is recognizable via the lack of a (dark) depletion zone at the interface, whose presence is indicative to the slow mode. These computations have been performed on a $2048 \times 2048$ rectangular grid. The former corresponds to $\sim 13000$ particles, or $118 \mu \mathrm{m} \times 118 \mu \mathrm{m}$ (assuming 1.1 $\mu \mathrm{m}$ particles), a size comparable to that of Skjeltorp's images. ${ }^{5}$ 

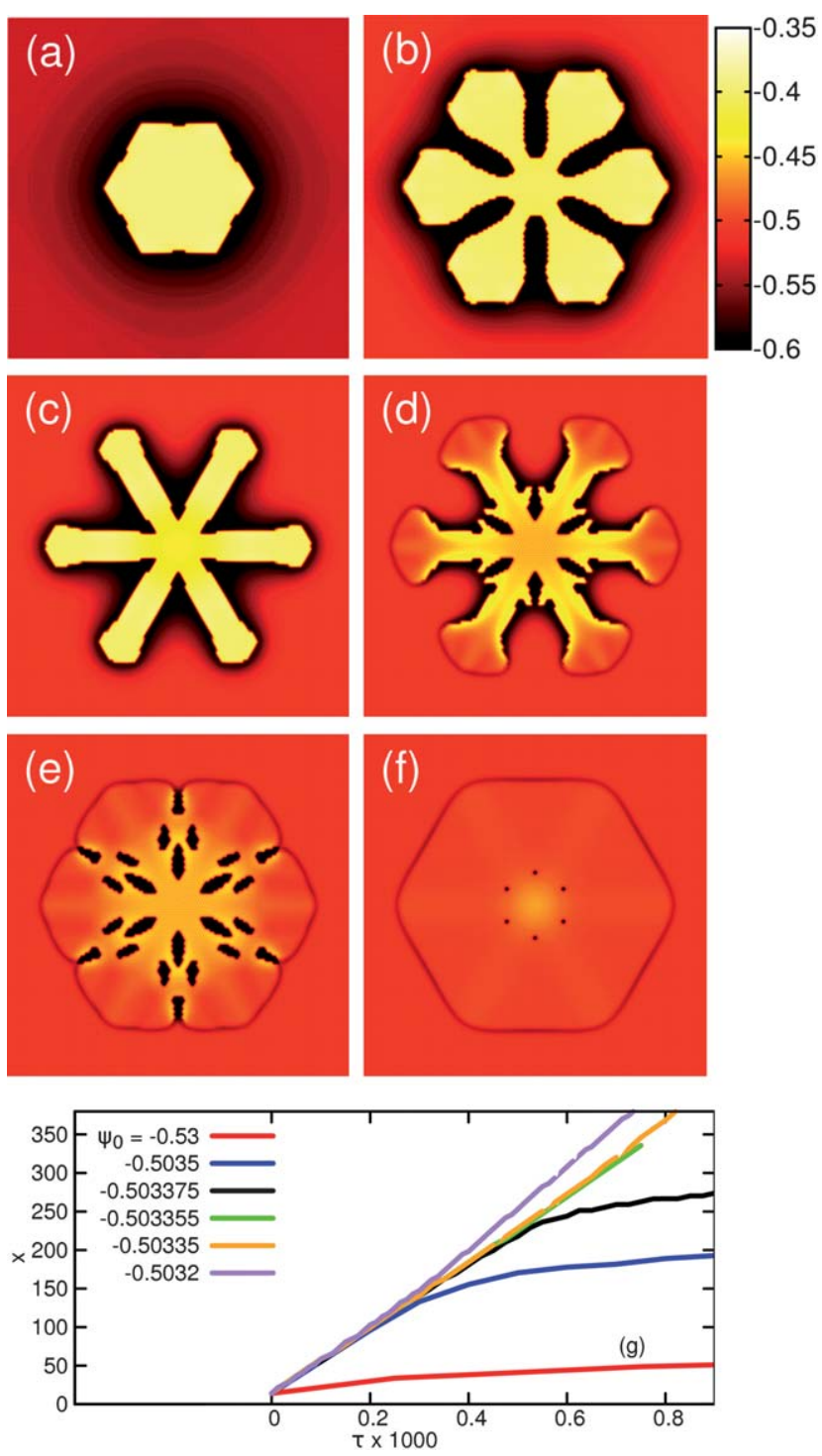

Fig. 2 Single crystal growth morphologies as predicted by the PFC model at $\varepsilon=-0.75$, while increasing reduced particle density $\left(\psi_{0}\right)$ in the absence of noise $(\alpha=0)$. The coarse-grained particle density map (=local average particle density, obtained by 2D FIR filtering, see text) is shown. Note the dark diffusion field ahead of the slowly growing faceted interfaces and the lack of such field at the fast growing broader interfaces. The reduced initial particle densities were as follows: (a) $\psi_{0}=-0.53$; (b) -0.5035 ; (c) -0.503375 ; (d) -0.503355 ; (e) -0.50335 ; and (f) -0.5032 . The computations have been performed on a $2048 \times 2048$ rectangular grid. (g) Dimensionless distance of the tip from the centre $v$ s. dimensionless time as a function of initial liquid density.

(Fig. 2b-d), and then by a fast-growing compact hexagonal morphology at high supersaturations (Fig. 2e and $\mathrm{f}$ ) having a broad non-faceted interface. In all these morphologies, the colloid particles are arranged on a 2D hexagonal crystal lattice. The change in growth anisotropy is also reflected in the rounding of corners with increasing driving force. Comparable morphological transitions are observed if the reduced particle density is kept constant and the temperature parameter $\varepsilon$ is changed (see Fig. 3); so this progressive change in morphology generally arises with increasing thermodynamic driving force through supersaturation or undercooling. What is the origin of these changes? A closer examination of the growth kinetics of noiseless growth patterns provides the necessary clues.

The kinetics of crystal growth is most basically monitored by simply tracking the overall size of the crystal growth pattern. We use the tip position of the crystal pattern to define the growth front position and the distance of this point from the growth centre of the crystal pattern to characterize the change in growth kinetics with thermodynamic driving force (Fig. 2g). For the 'near equilibrium' and low driving force case, we find a parabolic growth law $\left(x \propto \tau^{1 / 2}\right)$, while we find an essentially linear relationship $(x \propto$ $\tau)$ at high driving forces. For intermediate driving forces, there is initially a roughly constant growth velocity at first in a growth regime where the crystals have a compact shape, but this kinetics changes over to a continuously decreasing velocity in the regime where the faceted dendrites emerge. The crossover time separating these distinct growth regimes increases with increasing thermodynamic driving force.

\section{'Fast' and 'slow' growth modes}

We next examine the microscopic structure of the crystal-fluid interface at low and high driving forces to understand this
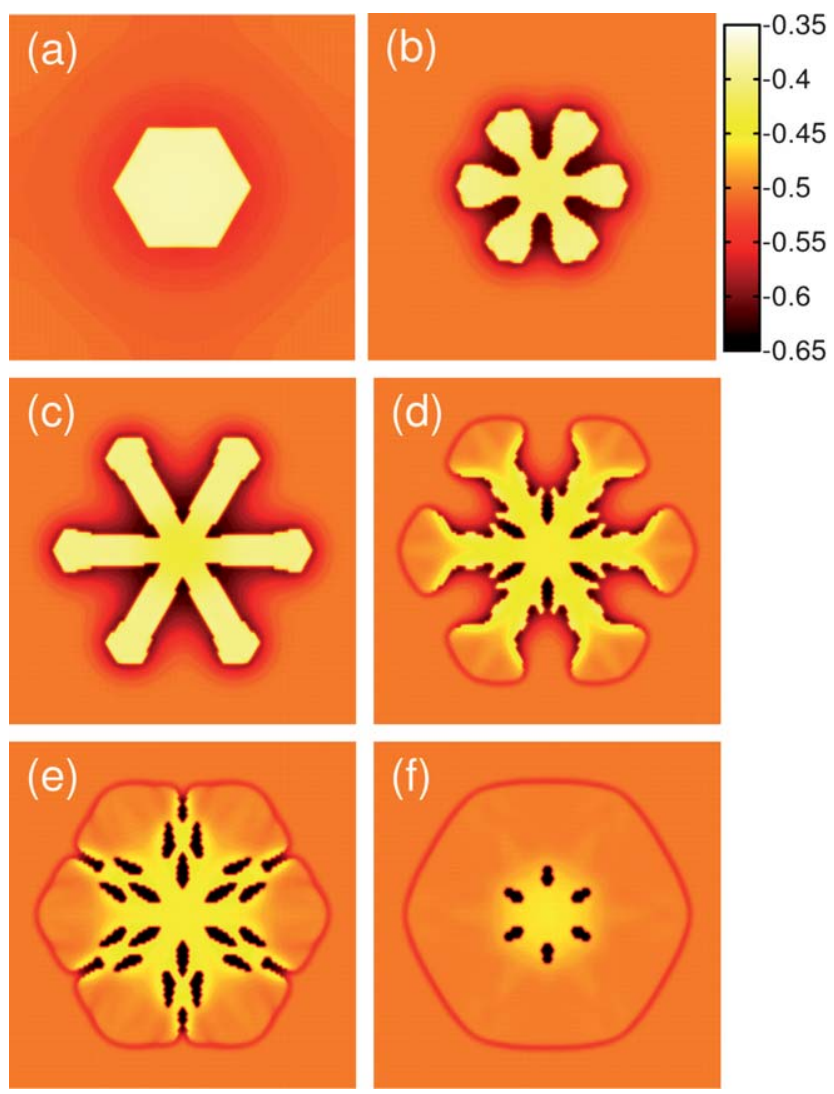

Fig. 3 Single crystal growth morphologies as predicted by the PFC model at $\psi_{0}=-0.503$, while decreasing the temperature in the absence of noise $(\alpha=0)$. The coarse-grained particle density map (=local average particle density, obtained by 2D FIR filtering, see text) is shown. Note the dark diffusion field ahead of the slowly growing faceted interfaces and the lack of such field at the fast growing broader interfaces. The respective values of the temperature parameter were as follows: (a) $\varepsilon=-0.6$; (b) -0.748 ; (c) -0.7488 ; (d) -0.74889 ; (e) 0.7489 ; and (f) -0.749 . The computations have been performed on a $2048 \times 2048$ rectangular grid. 
dramatic change in growth kinetics and crystal growth morphology. Some basic properties of the interfacial geometry under these variable growth conditions are summarized in Fig. 4. In particular, reduced particle density maps (the spatial variations of the local number density) are displayed in Fig. 4a and b, whereas the cross-interfacial variation of the coarse-grained particle density (the locally averaged particle density), $\tilde{\psi}$ is presented in Fig. $4 \mathrm{c}$ and d. The time evolution of the coarse-grained particle density field, $\tilde{\psi}$, across the interface is shown in Fig. $4 \mathrm{e}$ and $f$.

The faceted interface seen at low supersaturations is relatively sharp (Fig. 4a) and an extended density depletion zone builds up ahead of this growing front (Fig. $4 \mathrm{c}$ and e). This leads to crystal growth having a progressively decreasing velocity $\left(v \propto \tau^{-1 / 2}\right.$, see red curve in Fig. $2 \mathrm{~g}$ ), indicative of the front propagation being controlled by the rate of particle diffusion. This mode can be identified with the 'slow' mode described by Russel et al. ${ }^{7}$ and is also responsible for the appearance of the dendritic structures
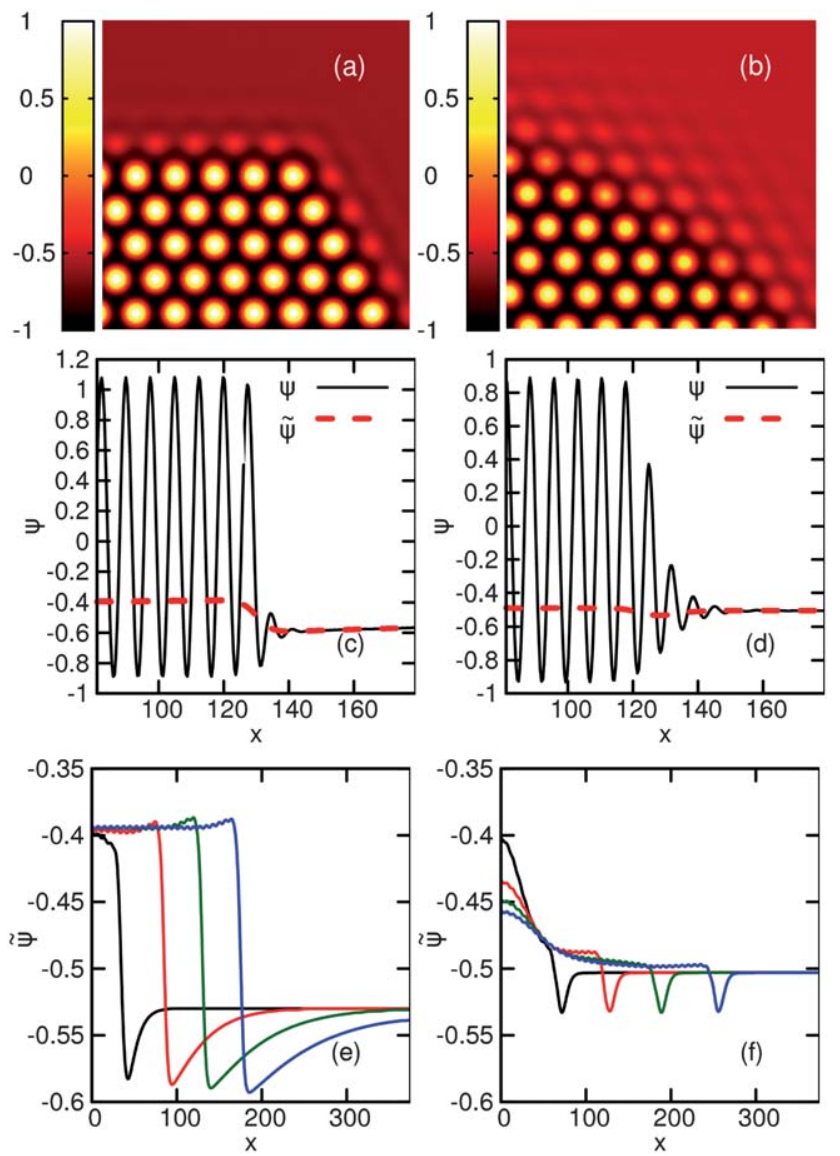

Fig. 4 Two modes of interface propagation: (a) faceted growth mode $\left(\psi_{0}=-0.53\right)$ with a sharp interface ("slow" mode); (b) non-faceted growth mode ( $\left.\psi_{0}=-0.5032\right)$ with a more diffuse interface ("fast" mode). (c and d) The local (solid) and the coarse-grained (dashed) particle density profiles at the growth front for the two modes. $x$ is the distance from the centre of the particle, along the horizontal centre line of the cluster. Note the broader interface for the fast mode and the higher density of the growing crystal for the slow mode. Time evolution of the coarse-grained density profile for (e) the slow and (f) the fast modes. Note the substantially reduced density difference between the solid and liquid phases in the latter case. shown in Fig. 2b-d where the same 'diffusive' growth kinetics is exhibited. These patterns represent a colloidal suspension analogue of the Mullins-Sekerka type diffusional instability leading to dendritic crystals in molecular fluids. ${ }^{35,36}$

In contrast to the sharp and faceted solid-liquid interface observed at near-equilibrium, a non-faceted interface that extends to several particle layers occurs (see Fig. $4 \mathrm{~b}$ and d) at high driving forces. This feature is accompanied by a weak, but well defined particle depletion zone that is localized near the solid-liquid interface (Fig. 4f). This distinct type of front propagates with a constant velocity (see grey line in Fig. 2 g). An essential clue into the physics underlying this relatively fast crystal growth mode is provided by Fig. $4 \mathrm{~d}$ and $\mathrm{f}$, which show that crystal crystals grown under these strong thermodynamic driving conditions have a substantially reduced density than those grown closer to equilibrium conditions. The crystal density somehow dynamically adjusts itself to be nearly equal to the initial particle concentration in the liquid! The net effect of this interfacial broadening is physically in line with the heuristic suggestion by Kyu and co-workers that increasing the thermodynamic driving force has the effect of a progressive reduction in the effective anisotropy of the interfacial free energy ${ }^{4}$ although this effect is not an equilibrium phenomenon in our simulations, as we discuss below.

The dramatic interfacial broadening and lower density crystal growth effect seem to be caused by the trapping of defects in the growing crystal. This dynamic density adjustment (trapping) mechanism in crystal growth is well established in the rapid solidification of alloys where this phenomenon is associated with solute trapping, ${ }^{37,38}$ making this mode of crystal growth an analogue of the fast or "diffusionless" crystal mode defined by Russel et al. ${ }^{7}$ We believe, furthermore, that this is the first demonstration of such a dynamic trapping of a conserved field in simulations based on microscopic theory. The next question is how these distinct slow and fast growth modes contribute to the progressive morphology changes seen in Fig. 1.

A basic difference in the slow and fast growth modes is their stability against diffusional instability induced finger growth. As noted before, interfaces growing by the slow growth mode are prone to the Mullins-Sekerka type instability, while the fast mode does not exhibit this type of interface branching instability. Remarkably, the two growth modes may coexist at the perimeter of the same single crystal (Fig. $2 \mathrm{~d}$ and e, and $3 \mathrm{~d}$ and e). The fast mode apparently has a regularizing effect on the growing dendrites in this regime of crystal growth, whereas the slow mode gives rise to the formation of sharp facets on the lateral sides of the dendrites. The evolving pattern structure with a change in driving force suggests that the local 'choice' between the two modes is caused by differences in the local crystallography (front orientation relative to crystal lattice). Progressive particle depletion ahead of the front starts to develop normally at the centre of faces, and the diffusive mode usually prevails once present. For weak depletion, however, the depleted zones remain localized and the crystal growth due to the fast mode is able to outrun this depletion front on both sides, leading to fluid pockets trapped into the solid. The frozen result of this phenomenon is apparent in the periodic pattern of crystal defects that can be clearly seen in the noise free crystal pattern in Fig. 2e and 3e, corresponding to fast mode crystal growth. 


\section{Density trapping}

We next verify the impression that the crystal density in the fast growth regime is approaching the particle density of the liquid so that there is so much defect in the crystal (excess vacancy concentration as the lattice constant does not seem to change perceptibly) that hardly any volume change occurs upon crystallization. If this effect is generally true for fast crystal growth, then its observation should provide a clear means of identifying fast mode crystal growth. We see in Fig. 5a that increasing the thermodynamic driving force for crystallization through an increase of the density causes the interface density to approach the initial density of the liquid. Next, we further quantify this interesting effect characterizing this peculiar fast mode growth.

The respective partition coefficient, defined in analogy to the partition coefficient in binary alloys, $k=\tilde{\psi}_{\text {sol }} / \tilde{\psi}_{\text {liq }}$, increases from $k_{0}=\psi_{\mathrm{S}} \mathrm{e} / \psi_{\mathrm{L}}{ }^{\mathrm{e}}=0.649$ towards 1 , where $\tilde{\psi}_{\text {sol }}$ and $\tilde{\psi}_{\text {liq }}$ are the reduced particle densities for the solid and liquid phases at the solidification front, while $\psi_{\mathrm{S}}{ }^{\mathrm{e}}=-0.4228$ and $\psi_{\mathrm{L}}{ }^{\mathrm{e}}=-0.6514$ are the coexisting solid and liquid densities. The PFC results for the velocity dependence of the partition coefficient and the theoretical models by Aziz, ${ }^{37} k=\left(k_{0}+v / v_{\mathrm{D}}\right) /\left(1+v / v_{\mathrm{D}}\right)$, and by Jackson et al. ${ }^{38} k=k_{0}{ }^{1 /\left(1+v / v_{\mathrm{D}}\right)}$, are in a qualitative agreement (Fig. $5 \mathrm{~b}$ ). Here $v_{\mathrm{D}}$ is the characteristic trapping velocity. In the range investigated, the PFC results can be fitted fairly well by $k_{0}=$ $0.641 \pm 0.002$ and $v_{\mathrm{D}}=0.159 \pm 0.004$ or by $k_{0}=0.641 \pm 0.002$ and $v_{\mathrm{D}}=0.128 \pm 0.003$, respectively, where the errors are standard deviations of the fitted parameters. The transition from the fully diffusion-controlled case $\left(v \ll v_{\mathrm{D}}\right)$ to the fully diffusionless case $\left(v \gg v_{\mathrm{D}}\right)$ is accompanied by about a two orders of magnitude increase in the crystal growth velocity, justifying the term 'fast' crystal growth mode.
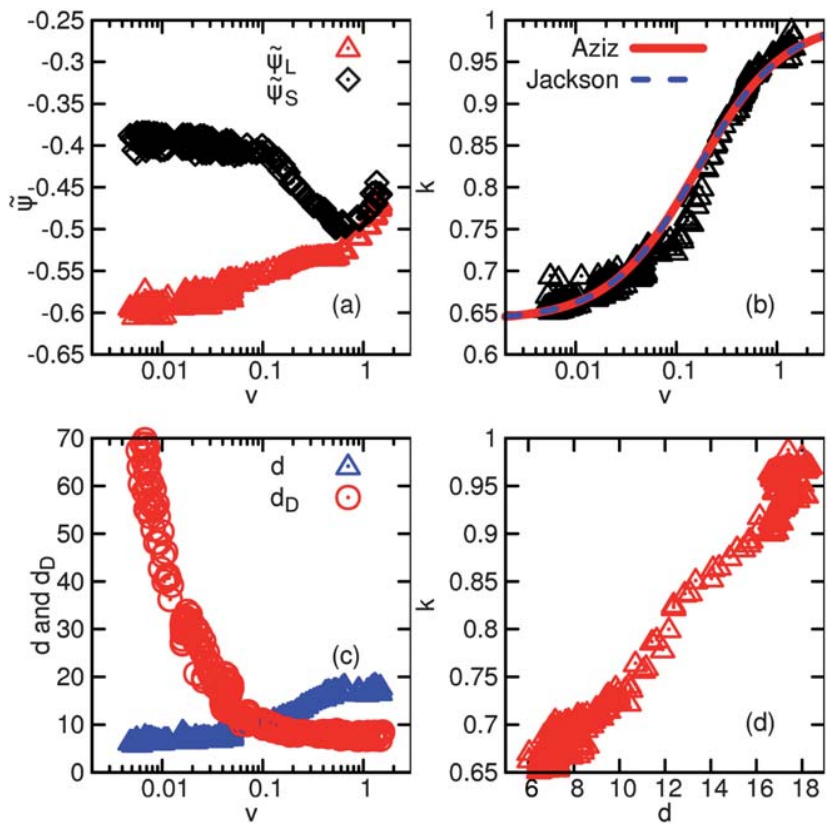

Fig. 5 Quantitative characterization of density trapping in the cases displayed in Fig. 1. (a) Coarse-grained solid and liquid densities at the growth front $v s$. velocity, $v$. (b) Partition coefficient, $k$, vs. velocity. (c) Interface thickness, $d$, and diffusion length, $d_{\mathrm{D}}$, vs. velocity. (d) Partition coefficient, $k, v s$. the interface thickness, $d$.

\section{Dynamic interface broadening}

Remarkably, there is also a dynamic broadening (also termed as kinetic roughening) of the interface region with increasing $v$ (see Fig. 5c), a phenomenon observed recently for colloidal systems. ${ }^{39}$ An interesting consequence of this dynamic interface broadening is a reduction of the growth anisotropy with increasing driving force (see Fig. 2-4). This phenomenon is analogous to the drastic reduction of the growth anisotropy due to kinetic roughening found in the interface dynamics of the Ising model ${ }^{40}$ and other critical systems. Such a relationship between the interface thickness and anisotropy is common, when approaching the critical point of a phase transition. (As in other models with a critical point, in the PFC model the interface thickness increases while the anisotropy decreases ${ }^{23,26,30}$ upon approaching the critical point). The usual rationale for this behaviour is that the properties of a broad interface are less sensitive to the relative orientation between the crystal and its surface than in the case of a sharp interface (see e.g. the isotropic broad-interface equilibrium crystallites near the critical point, and the hexagonal faceted crystals of a sharp interface far from it in Fig. 10).

The observed interfacial broadening is expected to enhance dynamic density adjustment (trapping), since broadening of the solid-liquid interface is known to yield enhanced solute trapping. ${ }^{41}$ Indeed, we see a roughly linear relationship between $k$ and $d$ (Fig. 5d). This scaling relation for $k$ should be useful in estimating the extent of dynamic interface broadening. As expected from theory, ${ }^{37,38}$ dynamic trapping of density becomes significant when the diffusion length $d_{\mathrm{D}}$ becomes comparable to the interface thickness $d$. (The diffusion length is defined as $d_{\mathrm{D}}=A / \Delta \psi=\left\{\int\left(\psi_{0}-\tilde{\psi}\right) \mathrm{d} n\right\} / \max \left(\psi_{0}-\tilde{\psi}\right)$, where $n$ is a spatial coordinate perpendicular to the solid-liquid interface. The integral represents the particle deficit ahead of the growth front, given by the area $A$ enclosed between the $\tilde{\psi}(n)$ and $\psi(n)=\psi_{0}$ curves, while $\Delta \psi=\max \left(\psi_{0}-\tilde{\psi}\right)$ is the maximum local depletion at the front relative to $\psi_{0}$.) Indeed, the intersection of the $d(v)$ and $d_{\mathrm{D}}(v)$ curves yields $v \approx 0.1$ (Fig. $5 \mathrm{c}$ ), which is comparable to the $v_{\mathrm{D}}$ data obtained by fitting the theoretical expressions to the observed partition coefficients. ${ }^{42}$

\section{Emergence of complex crystal growth forms}

These observations imply that 'fractal geometry' crystal growth forms arise as a consequence of a fluctuation-induced local selection between the fundamental slow and fast crystal growth modes. Initially, the crystallites grow with the fast mode at high thermodynamic driving force, but fluctuations disrupt the uniformity of the density distribution along the crystal perimeter, and then trigger an uneven particle depletion, which in turn breaks up the originally smooth front into fast travelling tips and slow diffusion-controlled regions. The tip trajectories become erratic due to the fluctuations, leading to fractal morphology. This fast mode branching instability evidently involves a distinct mechanism from the Mullins-Sekerka diffusive instability that applies for the slow diffusive mode of crystal growth.

The porous clusters form, when the fluctuation-induced defect trapping is prevalent and when noise representing the thermal fluctuations is also present (see Fig. 1). Under these conditions, 
the fast mode evidently does not have a regularizing effect of the shape of the crystal growth pattern, as in the symmetric crystals shown in Fig. 2e and $\mathrm{f}$, or $3 \mathrm{e}$ and $\mathrm{f}$. The morphologies in Fig. 1 show a striking resemblance to the experimental images by Skjeltorp ${ }^{5}$ of colloidal crystals grown under relatively low and high supersaturation in our simulations. The present model provides a unified description of these conspicuous changes in crystal morphology with thermodynamic driving force. As a further quantification of the morphological changes in Fig. 1 and to better compare with the observations by Skjeltorp, ${ }^{5}$ we determined the bulk fractal dimension $f_{\mathrm{d}}$ for the clusters shown in Fig. 1. The retrograde variation of $f_{\mathrm{d}}$ as a function of supersaturation nicely accords with Skjeltorp's estimates. ${ }^{5}$ The comparison with his measurements appears to be quantitative.

\section{Broader implications}

Finally, we discuss some of the broader implications of our results for understanding crystal growth in other materials. For our colloidal crystal growth study, the time evolution of the ordered crystal growth pattern (i.e., the particle density) follows diffusive dynamics. In a conventional coarse-grained phase-field type formulation, the situation addressed here corresponds to coupling of a non-conserved crystal-liquid order parameter to a coarse-grained particle density field, which follows diffusive dynamics. A similar situation occurs during the freezing of a binary alloy or in thermal transport controlled solidification, where the structural order parameter monitoring the crystalliquid transition is coupled either to the concentration field or the temperature field, both following diffusive dynamics. On the basis of the formal mathematical similarity of these problems we expect our results to be relevant to a broad class of crystallizing systems. For example, the progressive change of crystal morphology seen in Fig. 1-3 upon increasing the thermodynamic driving force can rationalize the change observed in the crystallization morphology of ultrathin polystyrene films from hexagonal crystals at low undercooling to symmetric dendrites and then progressively disordered dendrites ('seaweed') with decreasing crystallization temperatures. ${ }^{1,2}$ The latter structure is known to form only at small anisotropies (of both the kinetic coefficient and the interfacial free energy). A possible mechanism for achieving a low effective anisotropy is via forming a large number of randomly oriented crystallites at the growth front, as indeed happens in many cases. ${ }^{1,43}$ However, polycrystalline growth is apparently not the only mechanism of this morphology change. Taguchi et al. ${ }^{2}$ have shown that polystyrene crystals grown under similar conditions grow as single crystal, despite the increasingly irregular nature of the crystal growth upon increased cooling. The heuristic interpretation of $\mathrm{Xu}$ et al. ${ }^{4}$ of this change of crystal growth morphology with undercooling as arising from a progressive change in the surface tension anisotropy also has merit, although no mechanism accounting for such an explanation was provided in their work. The present paper provides the missing conceptual link: dynamic broadening of the interface indeed leads to the assumed change in the anisotropy of the interfacial free energy, while trapping of the relevant conserved quantity removes the propensity for branching. As pointed out earlier, the attendant growth of 'dynamic heterogeneity' (large dynamic mobility associated transient local ordering) in glass-forming and other complex fluids can give rise to a tendency towards disorderly polycrystalline growth ${ }^{1,43}$ and impurities ${ }^{44}$ can have a similar effect. These factors are important in the ultimate limit of this type of disorganized crystal growth, ${ }^{1,43}$ the polycrystalline spherulites, where the randomness of the nucleation process during the course of growth restores the isotropy of the crystallization growth pattern.

The present 'atomistic' field theoretic model of crystal growth is able to describe the observed progressive changes in growth morphology in colloidal crystal growth from compact symmetric crystal growth patterns to the formation of symmetric dendrites and finally disordered seaweed-like crystals at progressively large supersaturation. The same effect is seen upon lowering temperature so these morphology changes are characteristic of increasing the thermodynamic driving force for crystallization. By reducing the noise strength in our calculations, we are able to locate the essential cause of this morphology evolution with the strength of thermodynamic driving. We find that two distinct modes of crystal growth underlie the growth dynamics. First, there is the well-established 'slow' mode of crystal growth, which is subject to the well known Mullins-Sekerka instability: the crystal growing into the undercooled/supersaturated liquid develops fingers as part of its surface runs out ahead of the rest due to local interfacial fluctuations that initiate the process and the higher crystal growth rate engendered by the interface curvature. This type of diffusional instability is well known in molecular fluids and is the general origin of dendrite patterns forming in both colloidal fluids and fluid melts. ${ }^{7,12}$

Experiments on dendritic growth in a variety of systems indicate a progressive transformation of symmetric dendritic growth to irregularly shaped crystal patterns at high thermodynamic driving forces. We find this mode of crystal growth involves a 'fast' mode of crystal growth that occurs when crystal growth becomes too rapid to allow for the build-up of the diffusion field ahead of the crystal growth front. This 'fast' mode of crystal growth is normally much faster than the slow diffusive mode and is characterized by a crystal density that is similar to the particle density in the liquid. This means that the defect (vacancy) density within the resulting crystals is relatively high. The fast mode can mix with the slow mode in response to the time evolution of the particle depletion field ahead of the crystal growth front leading to significant effects on the crystal morphology: at relatively strong driving force conditions, there can be a stochastic switching between the fast and slow growth modes that can cause the crystal growth to take the form of highly branched 'fractal' structures, while retaining long range crystal order. Very similar crystal patterns have been seen in polymer films with increasing thermodynamic driving and we suggest that the same type of fast and slow mode competition is responsible for the morphology changes with driving force observed in these systems.

At extremely high undercooling or supersaturation, we expect these single crystal morphologies to ultimately give way to polycrystalline crystal growth due to lattice defects quenched into the solid that lead to the formation of new grains oriented differently at the growth front, as discussed in our previous work. ${ }^{1,43}$ Thus, strong thermodynamic driving ultimately restores the 'symmetric' nature of crystal growth pattern for both single crystal and polycrystalline growth forms. For single crystal growth, this terminal 
shape is determined by the kinetic anisotropy, while for polycrystalline growth the growth shape is isotropic (spherical in three dimensions or circular in near two dimensions), as the local growth anisotropy is randomized by the formation of grains with random orientation at interface of the growing crystal. At a late stage of this disorderly crystal growth, where this randomization effect becomes fully developed, the maximum growth rate direction of crystal growth is then along a direction perpendicular to the spherical envelope of the spherulitic polycrystal. ${ }^{1,43}$ Non-equilibrium crystal growth morphologies reflect the interplay between thermodynamic and kinetic anisotropies in the particle interactions that encode the ultimate crystal morphology, allowing for a significant control of morphology by tuning the growth conditions.

\section{Appendix A. Conditions for the PFC simulations}

The input parameters of the model can be combined into two independent dimensionless quantities, $\varepsilon$ and $\alpha$. These are dimensionless similarity parameters, such as the Reynolds or Pèclet numbers. Accordingly, a solution obtained for a single set $(\varepsilon, \alpha)$ corresponds to an infinite set of choices of the material properties/conditions. The magnitude of $\alpha$ is related to measurable quantities as follows: $\alpha=2\left(\rho_{\mathrm{L}}{ }^{\text {ref }} k T\right)^{2} /\left(3 K^{2} \rho_{\mathrm{L}}{ }^{\text {ref }} R^{\mathrm{d}}\right)$, where $K$ is the dimensional bulk modulus of the crystal, $d$ is the number of dimension, $\rho_{\mathrm{L}}{ }^{\text {ref }}$ is the number density of the reference liquid, while $R$ is the size-scale, i.e., the magnitude of $\alpha$ depends on $K$, $\rho_{\mathrm{L}}{ }^{\text {ref }}, T$, and $R$. Taking some typical values corresponding to the hard-disc system at a density of $\rho_{\mathrm{L}}{ }^{\text {ref }}=0.8$ and $\chi \rho_{\mathrm{L}}{ }^{\text {ref }} k T=0.06^{45}$ ( $K=1 / \chi$, where $\chi$ is the compressibility), one obtains $\alpha=$ $0.007 \approx 0.01$, close to the magnitude, we have used in our computations. This choice of $\alpha$ ensures also that on the timescale of the simulations we do not observe homogeneous nucleation. This is advantageous as we wish to study the large-scale growth forms observed by Skjeltorp. (At high nucleation rates, the impingement of a large number of small crystallites would prevent us from observing large-size growth forms.) The parameter $\varepsilon$ can be related to the expansion coefficients of the two-particle direct correlation function, and via them to the compressibility of the liquid and the bulk modulus of the solid. Considering these relationships, and Hall's equations of state for the fluid and crystalline phases of the hard-sphere system, ${ }^{46}$ one obtains $\varepsilon \approx-0.45$ to -0.56 in the density range of crystal-liquid coexistence. This indicates that the hard-sphere colloidal systems fall farther from the critical point than the noble gas $\mathrm{Ar}$, characterized by $\varepsilon \approx$ -0.13 to -0.27 deduced by Elder and Grant. ${ }^{17}$ To our understanding, the relevant data for Skjeltorp's experimental 2D system are not available therefore we cannot make a similar estimate. The faceting observed in experiments implies a large negative value for $\varepsilon$. We note that the qualitative behaviour is very similar for $\varepsilon \approx$ -0.5 and -0.75 , thus we believe our choice is reasonable for qualitative modelling of colloidal systems. A recent work has explored the behaviour of the PFC model at far larger distances from the critical point, and interpreted the PFC approach in this range as a reasonable model of foams. ${ }^{47}$

\section{Appendix B. The PFC phase diagram in 2D}

In 2D, the deterministic (zero noise strength) PFC model predicts the following stable phases: ${ }^{17}$ a homogenous disordered (fluid) phase, an ordered hexagonal (crystalline) phase, and a striped phase (see Fig. 6). The state points at which our simulations have been performed and the relevant phase boundaries are indicated in Fig. 6 in reduced temperature and particle density units. The deterministic Brazovskii/Swift-Hohenberg/PFC models (which are equivalent so far as the equilibrium properties are concerned) lead to mean-field critical exponents ${ }^{24}$ that are independent of dimensionality. Results from deterministic PFC simulations for the interface thickness and line tension in 2D are consistent with this. ${ }^{30}$ Adding noise to the equation of motion is expected to change the position of the critical point and to drive the system into different universality classes depending now on dimensionality. It has been shown by Brazovskii using the self-consistent Hatree approximation that the fluctuations destroy the meanfield instability and lead to a weakly first-order transition ${ }^{\mathbf{1 9 2 0 , 4 8}}$ as confirmed by more accurate renormalization calculations. ${ }^{49}$

\section{Appendix C. Melting and freezing in 2D in the PFC model}

\section{Melting}

According to Kosterlitz, Thouless, Halperin, Nelson, and Young, melting of 2D crystals happens in two steps by second order phase transitions at two distinct temperatures. In the first step, melting is driven by thermally activated dislocation pairs that dissociate and transform the crystal into an orientationally ordered hexatic phase, while in the second step this orientational order is destroyed by dissociation of free dislocations into disclinations that drives the system into the disordered liquid state. ${ }^{33}$ Computer simulations for the hard-disc system seem to support the prediction that melting in $2 \mathrm{D}$ systems occurs as a two-stage process ${ }^{33}$ first, there is a transition from a crystal to a hexatic phase having long range orientational correlations and then to a liquid phase where the local orientational correlations are lost. However, there is no consensus on the order of these transitions. Some works indicate a continuous transition for the first stage and a first-order transition for the second stage, while others claim the situation is the other way around. While the situation regarding melting is not fully resolved, we can make some comments on this phenomenon based on our PFC simulations.

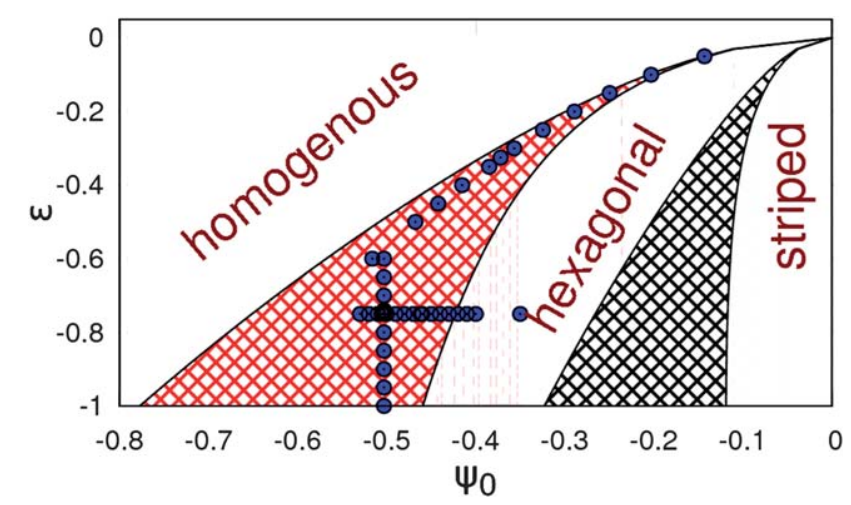

Fig. 6 The $\psi<0$ section of the $\mathrm{B} / \mathrm{SH} / \mathrm{PFC}$ phase diagram predicted in 2D using the single-mode approximation. ${ }^{17}$ The blue circles denote the points in which simulations have been performed. Note the critical point at $\psi_{\mathrm{c}}=0$ and $\varepsilon_{\mathrm{c}}=0$. 
There is no indication for the formation of the hexatic phase in the PFC model. This might be viewed as a possible weakness of the model. However, the hexatic phase has been found elusive both experimentally and in simulations: ${ }^{33}$ apparently finite-size effects and the type of interaction potential influence its appearance, some studies imply that it is metastable, and it is worth noting that the validity of the Mermin-Wagner theorem that excludes the long-range crystalline order in $2 \mathrm{D}$ is restricted to only non-hard core pair-potentials, ${ }^{50,51}$ i.e., we cannot a priori exclude that an infinite $2 \mathrm{D}$ crystalline phase may exist both in hard-disc type colloids and in the PFC model. (We stress that the present paper addresses crystalline aggregation after quenching, not melting close to equilibrium.) Whether the hexatic phase can be observed in near-equilibrium studies of melting within the PFC model is an open question. The hexatic phase might remain unobservable if the time averaging inherent in the PFC washes out the structural features so that the hexatic phase is essentially indistinguishable from the fluid phase.

Our preliminary simulations for near equilibrium melting suggest the nucleation of melting via vacancy generation for single crystals and grain boundary melting for the polycrystalline state (Fig. 7). This accords with results, obtained with a hardcore repulsive interaction. ${ }^{52}$

\section{Freezing}

In experiments on 2D colloids, freezing after quenching occurs by direct nucleation of the $2 \mathrm{D}$ crystallites without the appearance of the hexatic phase, ${ }^{31}$ signalling a first-order transition. In other

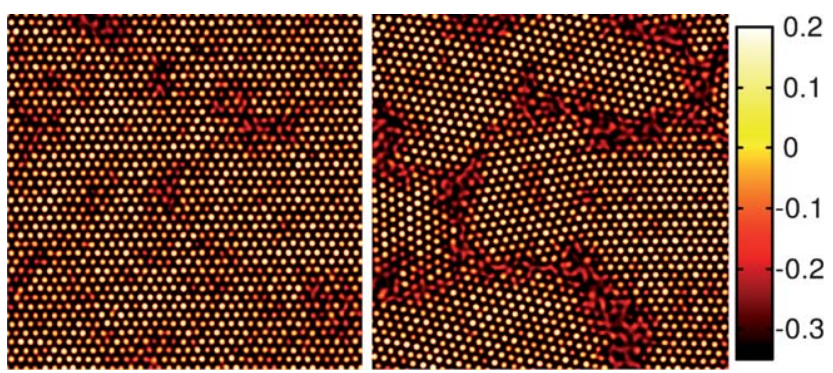

Fig. 7 Melting in the PFC model. Initial state: single crystal (left) and polycrystalline state (right). $\left(\varepsilon=-0.25, \alpha=0.1, \psi_{0}=-0.27\right.$, a $1452 \times$ 1452 fraction of a $4840 \times 2092$ grid is shown.)
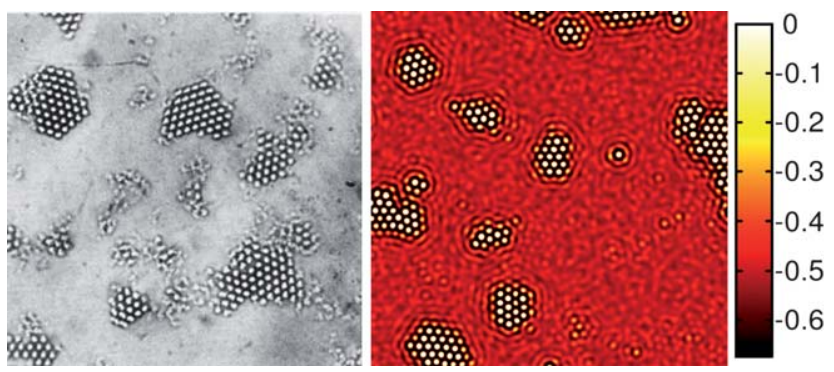

Fig. 8 Homogeneous crystal nucleation and growth in $2 d$ colloidal systems at relatively high supersaturation. Left: experiment, ${ }^{15}$ with the permission of the APS; $\odot$ APS 1985; right: PFC simulation with noise. $(\varepsilon=$ $-0.75, \alpha=0.012, \psi_{0}=-0.5038$, a $1495 \times 1495$ fraction of a $2048 \times 2048$ grid is shown.) works, 2D crystal nucleation happens by a two-step process via an amorphous precursor. ${ }^{32}$ The present model recovers this complex behaviour, ${ }^{23,30}$ specifically, at small undercoolings direct crystal nucleation is observed from the fluid, while at the large undercoolings and noise strengths, we observe the formation of an amorphous phase preceding the formation of the crystal (and as in the experiments the spatial decay of the bond-order correlation function excludes the appearance of the hexatic phase ${ }^{23}$ ).

At the noise strength, we have chosen for the present study, there is no homogeneous nucleation on the timescale of the simulations. Alternatively, we can choose the noise strength so that on the timescale of the simulation we see nucleation events. Indeed, with increased noise strength (e.g., $\alpha=0.1$ ), we see the homogeneous nucleation of the crystalline phase, and the patterns forming have again a striking similarity to the experiments (Fig. 8). At lower supersaturations one needs larger noise strength to observe nucleation on the timescale accessible for simulations. However, the time evolution of morphology from chain-like aggregates to faceted crystallites follows the sequence seen in experiments (Fig. 9).

\section{Appendix D. Growth anisotropy, equilibrium shapes and anisotropy of the interfacial free energy}

Growth anisotropy occurs as interplay of several factors: (i) anisotropy of the interfacial free energy, (ii) anisotropy of the

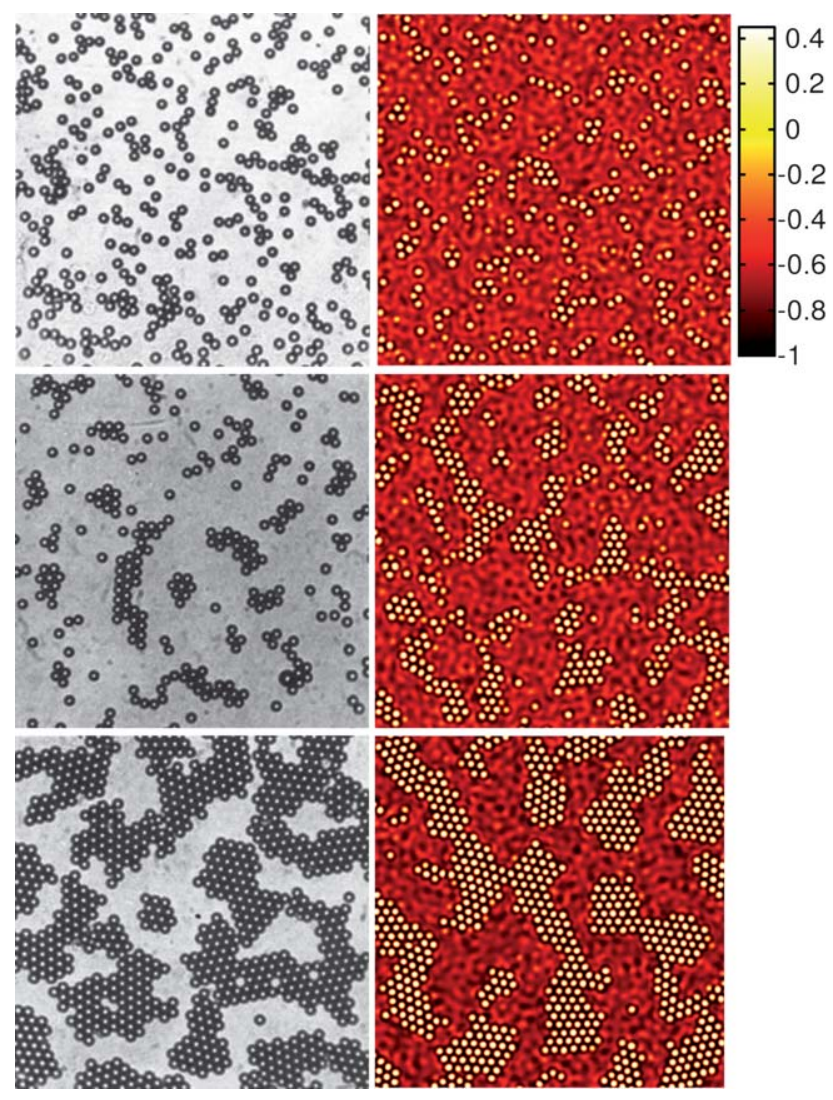

Fig. 9 Homogeneous crystal nucleation and growth in $2 \mathrm{~d}$ colloidal systems at reduced supersaturation. Left: experiment, ${ }^{15}$ with the permission of the APS; (C) APS 1985; right: PFC simulation with noise. $(\varepsilon=$ $-0.75, \alpha=0.5, \psi_{0}=-0.6$, a $733 \times 733$ fraction of a $1024 \times 1024$ grid is shown.) 
kinetic coefficient that describes the attachment rate of particles to the crystal, and (iii) diffusional instabilities.

The equilibrium shapes (that minimise the contribution from the interfacial term to the free energy to a $2 \mathrm{D}$ crystal of given area) have been obtained by placing a seven particle cluster into the simulation box and letting it grow until establishing equilibrium (see Fig. 10). The initial liquid density has been chosen so that the expected crystalline fraction obtained from the lever rule is $X=0.3$. As expected from computer simulations and various theoretical treatments, ${ }^{23,26,30,53,54}$ the interface thickness diverges at the critical point in Fig. 6, while the anisotropy increases with increasing distance from the critical point.
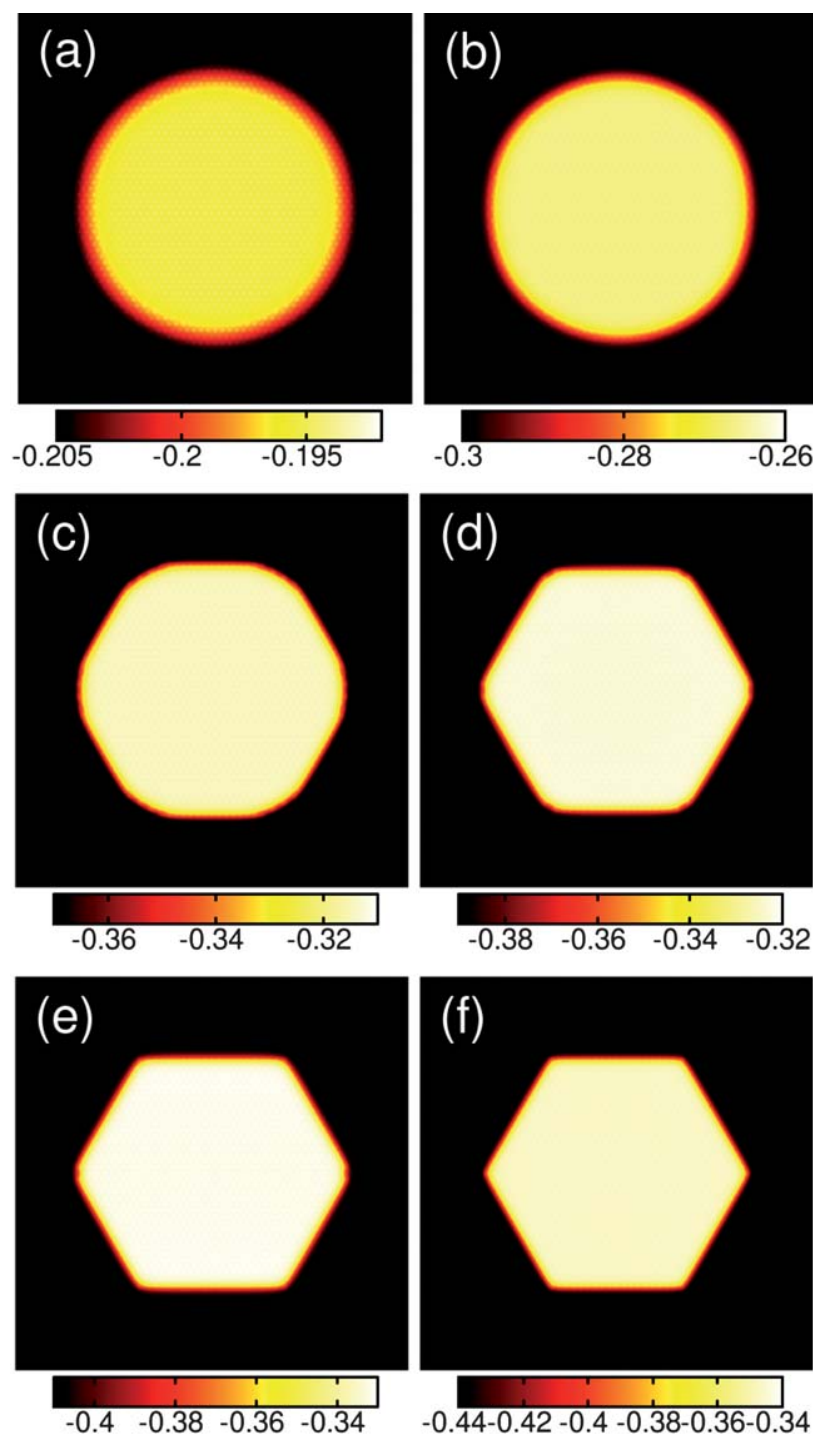

Fig. 10 Equilibrium shape $v$ s. temperature $(\varepsilon)$ as predicted for a crystalline fraction of $X \approx 0.3$ (see the respective points in Fig. 6). (a) $\varepsilon=$ -0.1 ; (b) -0.2 ; (c) -0.3 ; (d) -0.325 ; (e) -0.35 ; and (f) -0.4 . The coarsegrained (FIR filtered) reduced particle density maps are shown. Note that the interface thickness decreases while the anisotropy increases with an increasing distance from the critical point (from left to right). The computations have been performed on a $1024 \times 1024$ rectangular grid. Equilibration has been performed in a period of $10^{6}$ dimensionless time steps.
The anisotropy of the equilibrium interfacial free energy predicted by the PFC model has been discussed extensively in previous work. ${ }^{17,23-26,30}$ It has also been shown in a multiscale analysis relying on a small- $\varepsilon$ expansion of the free energy that the anisotropy of the interfacial free energy originates from different cross-interfacial variations of the amplitudes for the dominant Fourier modes (density waves), whose wave vectors are among the first reciprocal lattice vector star but have different orientational relationship to the interface normal. ${ }^{24,25}$ These results refer to the deterministic case (zero noise strength). In this case, the Brazovskii/Swift-Hohenberg/PFC models (which are equivalent so far as the equilibrium properties are concerned) lead to meanfield critical exponents ${ }^{24}$ that are independent of dimensionality. Our noiseless numerical results obtained for the interface thickness and line tension in $2 \mathrm{D}$ are consistent with this result. ${ }^{30}$

As the PFC method is a true atomistic model (DFT), the numerical simulations automatically incorporate factors (i)-(iii), besides, elasticity, surface reconstruction, etc. As a result, similarly to other atomistic approaches as molecular dynamics, separation of the individual effects is difficult. Regarding these difficulties we are left with general considerations, when interpreting growth anisotropy, such as the observation that a broad interface is more forgiving to the relative orientation between the interface and the crystal than an interface sharp on the atomistic scale, a phenomenon demonstrated by simulations within the $2 \mathrm{D}$ Ising model. ${ }^{40}$

\section{Acknowledgements}

This work has been supported by the EU FP7 Collaborative Project ENSEMBLE under Grant Agreement NMP4-SL-2008213669 and by the Hungarian Academy of Sciences under contract OTKA-K-62588.

\section{Notes and references}

1 L. Gránásy, T. Pusztai, T. Börzsönyi, J. A. Warren and J. F. Douglas, Nat. Mater., 2004, 3, 645-650.

2 K. Taguchi, H. Mayaji, K. Izumi, A. Hoshino, Y. Miyamoto and R. Kokawa, Polymer, 2001, 42, 7443-7447.

3 K. L. Beers, J. F. Douglas, E. J. Amis and A. Karim, Langmuir, 2003, 19, 3935-3940.

4 H. J. Xu, R. Matkar and T. Kyu, Phys. Rev. E: Stat., Nonlinear, Soft Matter Phys., 2005, 72, 0110804.

5 A. T. Skjeltorp, Phys. Rev. E: Stat. Phys., Plasmas, Fluids, Relat. Interdiscip. Top., 1987, 58, 1444-1447.

6 B. J. Ackerson and K. Schätzel, Phys. Rev. E: Stat. Phys., Plasmas, Fluids, Relat. Interdiscip. Top., 1995, 52, 6448-6366.

7 W. B. Russel, P. M. Chaikin, J. Zhu, W. V. Meyer and R. Rogers, Langmuir, 1997, 13, 3871-3881.

8 P. Sandkühler, M. Lattuada, H. Wu, J. Sefcik and M. Morbidelli, Adv. Colloid Interface Sci., 2005, 113, 65-83.

9 R. Wild and P. Harrowell, J. Chem. Phys., 2001, 114, 9059-9068.

10 J. F. Lutsko, Phys. Rev. E: Stat., Nonlinear, Soft Matter Phys., 2006, 74, 021121

11 S. van Teeffelen, C. N. Likos and H. Löwen, Phys. Rev. Lett., 2008, 100, 108302.

12 Z. Cheng, P. M. Chaikin, J. Zhu, W. B. Russel and W. V. Meyer, Phys. Rev. Lett., 2002, 88, 015501.

13 P. Pieranski, Phys. Rev. Lett., 1980, 45, 569-572.

14 A. J. Hurd and D. W. Scheafer, Phys. Rev. Lett., 1985, 54, 1043-1046.

15 G. Y. Onoda, Phys. Rev. Lett., 1985, 55, 226-229.

16 K. R. Elder, M. Katakowski, M. Haataja and M. Grant, Phys. Rev. Lett., 2002, 88, 245701.

17 K. R. Elder and M. Grant, Phys. Rev. E: Stat., Nonlinear, Soft Matter Phys., 2004, 70, 051605. 
18 T. V. Ramakrishnan and M. Yussouff, Phys. Rev. B: Condens. Matter, 1979, 19, 2775-2794.

19 S. A. Brazovskii, Zh. Eksp. Teor. Fiz., 1975, 68, 175-185.

20 J. Swift and P. C. Hohenberg, Phys. Rev. A: At., Mol., Opt. Phys. 1977, 15, 319-328.

21 J. Berry, K. R. Elder and M. Grant, Phys. Rev. E: Stat., Nonlinear, Soft Matter Phys., 2008, 77, 061506.

22 G. Tegze, G. Bansel, G. I. Tóth, T. Pusztai, Y. Fan and L. Gránásy, J. Comput. Phys., 2009, 228, 1612-1623.

23 L. Gránásy, M. Tegze, G. I. Tóth and T. Pusztai, Philos. Mag., 2011 91, 123-149.

24 K.-A. Wu and A. Karma, Phys. Rev. B: Condens. Matter Mater. Phys., 2007, 76, 184107.

25 S. Majaniemi and N. Provatas, Phys. Rev. E: Stat., Nonlinear, Soft Matter Phys., 2009, 79, 011607.

26 R. Backofen and A. Voigt, J. Phys.: Condens. Matter, 2009, 21, 464109.

27 K. R. Elder, N. Provatas, J. Berry, P. Stefanovic and M. Grant, Phys. Rev. B: Condens. Matter Mater. Phys., 2007, 75, 064107.

28 T. Pusztai, G. Tegze, G. I. Tóth, L. Környei, G. Bansel, Y. Fan and L. Gránásy, J. Phys.: Condens. Matter, 2008, 20, 404205.

29 G. Tegze, L. Gránásy, G. I. Tóth, F. Podmaniczky, A. Jaatinen, T. Ala-Nissila and T. Pusztai, Phys. Rev. Lett., 2009, 103, 035702.

30 G. I. Tóth, G. Tegze, T. Pusztai, G. Tóth and L. Gránásy, J. Phys. Condens. Matter, 2010, 22, 364101.

31 P. Dillmann, G. Maret and P. Keim, J. Phys.: Condens. Matter, 2008, 20, 404216.

32 T. J. Zhang and X. Y. Liu, J. Am. Chem. Soc., 2007, 129, 13520 13526.

33 For a recent review see: H. H. Grünberg, P. Keim and G. Maret, in Soft Matter, Vol. 3: Colloidal Order From Entropic and Surface Forces, ed. G. Gompper and M. Schick, Wiley-VCH Verlag GmbH \& Co. KGaA, Weinheim, 2007, pp. 40-83.

34 R. L. Davidchack and B. B. Laird, J. Chem. Phys., 1998, 108, 9452 9462

35 W. W. Mullins and R. F. Sekerka, J. Appl. Phys., 1964, 35, 444-451.

36 Dendritic solidification evolving as interplay of the Mullins-Sekerka instability with crystal anisotropy normally happens with a constant velocity established after a brief initial transient.
37 M. J. Aziz, J. Appl. Phys., 1982, 53, 1158-1168.

38 K. A. Jackson, K. M. Beatty and K. A. Gudgel, J. Cryst. Growth, 2004, 271, 481-494.

39 R. P. A. Dullens, D. G. A. L. Aarts and W. K. Kegel, Phys. Rev. Lett., 2006, 97, 228301.

40 V. A. Shneidman, K. A. Jackson and K. M. Beatty, J. Cryst. Growth, 2000, 212, 564-573.

41 N. A. Ahmad, A. A. Wheeler, W. J. Boettinger and G. B. McFadden, Phys. Rev. E: Stat. Phys., Plasmas, Fluids, Relat. Interdiscip. Top., 1998, 58, 3436-3450.

42 Note that dynamic trapping sets in at the velocity $v_{\mathrm{D}}$ at which the diffusion length becomes comparable to interface thickness: $d_{\mathrm{D}}=$ $D / v_{\mathrm{D}} \approx d$ leading to $v_{\mathrm{D}} \approx D / d$. With typical data of metallic liquids $\left(D \approx 10^{-9} \mathrm{~m}^{2} \mathrm{~s}^{-1}\right.$ and $d \approx 1 \mathrm{~nm}$ ), the critical velocity is about $1 \mathrm{~m}$ $\mathrm{s}^{-1}$, while for colloids it is orders of magnitude smaller.

43 L. Gránásy, T. Pusztai, G. Tegze, J. A. Warren and J. F. Douglas, Phys. Rev. E: Stat., Nonlinear, Soft Matter Phys., 2005, 72, 011605.

44 L. Gránásy, T. Pusztai, J. A. Warren, J. F. Douglas, T. Börzsönyi and V. Ferreiro, Nat. Mater., 2003, $2,92$.

45 M. Brunner, C. Bechinger, U. Herz and H. H. von Grünberg, Europhys. Lett., 2003, 63, 791-797.

46 K. R. Hall, J. Chem. Phys., 1972, 57, 2252-2254.

47 N. Guttenberg, N. Goldenfeld and J. Dantzig, Phys. Rev. E: Stat., Nonlinear, Soft Matter Phys., 2010, 81, 065301(R).

48 G. H. Fredrickson and K. Binder, J. Chem. Phys., 1989, 91, 72657275.

49 D. D. Ling, B. Friman and L. P. Finn, Phys. Rev. B: Condens. Matter, 1981, 24, 2718-2730.

50 N. D. Mermin, Phys. Rev., 1968, 176, 250-254.

51 A. Gelfert and W. Noltig, J. Phys.: Condens. Matter, 2001, 13, R505R524.

52 P. Karnchanaphanurach, B. Lin and S. A. Rice, Phys. Rev. E: Stat. Phys., Plasmas, Fluids, Relat. Interdiscip. Top., 2000, 61, 4036-4044.

53 J. D. Gunton, M. San Miguel and P. Sahni, in Phase Transitions and Critical Phenomena, ed. C. Domb and J. L. Lebowitz, Academic, London, 1983, vol. 8, pp. 267-466.

54 For example: K. K. Mon, S. Wansleben, D. P. Landau and K. Binder, Phys. Rev. Lett., 1998, 60, 708-711; E. Bittner, A. Nussbaumer and W. Janke, Nucl. Phys. B, 2009, 820, 694-706. 